\title{
HaloTag is an effective expression and solubilisation fusion partner for a range of fibroblast growth factors
}

Changye Sun, Yong Li, Sarah E Taylor, Xianqing Mao, Mark C Wilkinson, David G Fernig

The production of recombinant proteins such as the fibroblast growth factors (FGFs) is the key to establishing their function in cell communication. The production of recombinant FGFs in E.coli is limited, however, due to expression and solubility problems. HaloTag has been used as a fusion protein to introduce a genetically-encoded means for chemical conjugation of probes. We have expressed 11 FGF proteins with an N-terminal HaloTag, followed by a tobacco etch virus (TEV) protease cleavage site to allow release of the FGF protein. These were purified by heparin-affinity chromatography, and in some instances by further ion-exchange chromatography. It was found that HaloTag did not adversely affect the expression of FGF1 and FGF10, both of which expressed well as soluble proteins. The $\mathrm{N}$-terminal HaloTag fusion was found to enhance the expression and yield of FGF2, FGF3 and FGF7. Moreover, whereas FGF6, FGF8, FGF16, FGF17, FGF20 and FGF22 were only expressed as insoluble proteins, their $\mathrm{N}$-terminal HaloTag fusion counterparts (Halo-FGFs) were soluble, and could be successfully purified. However, cleavage of Halo-FGF6, -FGF8 and -FGF22 with TEV resulted in aggregation of the FGF protein. Measurement of phosphorylation of p42/44 mitogen-activated protein kinase and of cell growth demonstrated that the HaloTag fusion proteins were biologically active. Thus, HaloTag provides a means to enhance the expression of soluble recombinant proteins, in addition to providing a chemical genetics route for covalent tagging of proteins. 
HaloTag is an effective expression and solubilisation fusion partner for a range of fibroblast growth factors

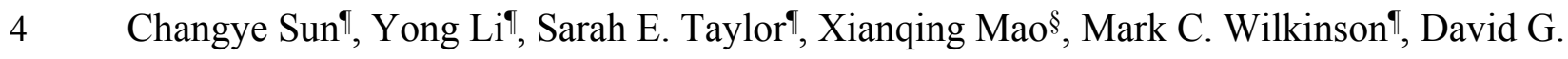

6 Department of Biochemistry, Institute of Integrative Biology, University of Liverpool, 7 Liverpool, UK, L69 7ZB; § Department of Oncology, Laboratory of Cellular and Molecular 8 Oncology, Luxembourg Institute of Health, 84 Val Fleuri, L-1526 Luxembourg. 


\section{Abstract}

10 The production of recombinant proteins such as the fibroblast growth factors (FGFs) is the key to establishing their function in cell communication. The production of recombinant FGFs in 12 E.coli is limited, however, due to expression and solubility problems. HaloTag has been used as 13 a fusion protein to introduce a genetically-encoded means for chemical conjugation of probes. 14 We have expressed 11 FGF proteins with an N-terminal HaloTag, followed by a tobacco etch virus (TEV) protease cleavage site to allow release of the FGF protein. These were purified by heparin-affinity chromatography, and in some instances by further ion-exchange chromatography. It was found that HaloTag did not adversely affect the expression of FGF1 and FGF10, both of which expressed well as soluble proteins. The N-terminal HaloTag fusion was found to enhance the expression and yield of FGF2, FGF3 and FGF7. Moreover, whereas FGF6, FGF8, FGF16, FGF17, FGF20 and FGF22 were only expressed as insoluble proteins, their Nterminal HaloTag fusion counterparts (Halo-FGFs) were soluble, and could be successfully purified. However, cleavage of Halo-FGF6, -FGF8 and -FGF22 with TEV resulted in aggregation of the FGF protein. Measurement of phosphorylation of p42/44 mitogen-activated protein kinase and of cell growth demonstrated that the HaloTag fusion proteins were biologically active. Thus, HaloTag provides a means to enhance the expression of soluble recombinant proteins, in addition to providing a chemical genetics route for covalent tagging of proteins. 


\section{Introduction}

Of the 18 receptor-binding fibroblast growth factors (FGF), 15 also bind a heparan sulfate coreceptor and are classed as growth factors and morphogens. These are grouped into 5 subfamilies based on their protein sequence similarity (Itoh 2007; Ornitz 2000), and they regulate a myriad of processes in development, homeostasis and in some diseases (Beenken \& Mohammadi 2009; Turner \& Grose 2010). Recombinant FGFs provide a key tool to study their structure-function relationships, and labelling FGFs for microscopy has been important in probing the mechanisms of, for example, their transport (Duchesne et al. 2012; Lin 2004; Yu et al. 2009). Chemical labelling has disadvantages compared to genetically encoded labelling, since with the latter it is easier to predict the structural and hence functional consequences of labelling, which can be achieved both in vitro and in vivo. While fluorescent proteins remain a mainstay of genetic labelling, they have limitations. These have been overcome, for example, by non-covalent tagging of proteins on hexahistidine sequences with Tris- $\mathrm{Ni}^{2+}$ nitriloacetic acid (Huang et al. 2009; Lata et al. 2005; Tinazli et al. 2005), which has allowed diverse labelling strategies, ranging from fluorescent dyes (Uchinomiya et al. 2009) and quantum dots (Roullier et al. 2009; Susumu et al. 2010) to gold nanoparticles (Duchesne et al. 2008). However, non-covalent coupling is reversible and exchange may occur in this instance with histidine-rich patches on endogenous proteins.

HaloTag is a mutant of a bacterial haloalkane dehalogenase, which reacts with chloroalkane ligands to form a covalent bond that represents the covalent intermediate of the enzyme's normal catalytic cycle (Los et al. 2008). Fluorescent dyes (Los et al. 2008) and quantum dots (Zhang et al. 2006b) carrying a chloroalkane group have been used to label HaloTag fusion proteins for fluorescence imaging. This approach is particularly versatile, since it combines the power of a genetically encoded tag (the HaloTag protein) with covalent labelling.

Consequently, we set out to produce N-terminal HaloTag fusions of different FGFs. In the course of this work, we observed that the N-terminal HaloTag fusion had a substantial effect on the expression of the more recalcitrant FGFs, consistent with the observation that HaloTag is a potential solubilisation tag for recombinant proteins (Ohana et al. 2009). Thus, whereas expression of FGF1 and FGF10 was somewhat reduced and that of FGF2 increased, expression of FGF7, which can be toxic (Ron et al. 1993), was no longer so, while expression of soluble FGF3, FGF6, FGF7, FGF8, FGF16, FGF17, FGF20 and FGF22 was markedly enhanced. This is in contrast to previous reports where FGFs such as FGF6 (Pizette et al. 1991), FGF8 (Loo \& Salmivirta 2002; Macarthur et al. 1995; Vogel et al. 1996), FGF16 (Danilenko et al. 1999) and FGF20 (Jeffers et al. 2002; Kalinina et al. 2009), have been found to be mainly expressed in inclusion bodies, even as truncated proteins, and so require refolding. Thus, HaloTag provides not just a means to label proteins covalently and specifically, but is also a useful solublisation partner for the production of recombinant proteins. 
70

71

72

73

74

75

76

77

78

79

80

81

82

83

\section{Materials}

pET-14b vectors containing cDNAs encoding FGF1 and FGF2 and pET-M11 vector containing

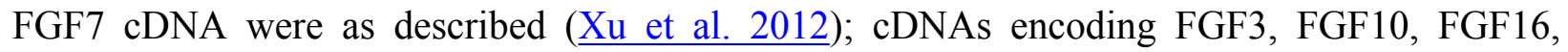
FGF17 and FGF20 were purchased from Eurofins Genomics (Ebersberg, Germany); cDNAs encoding FGF6, FGF8 and FGF22 were purchased from Life Technologies (Paisley, UK); cDNAs encoding HaloTag was acquired from Kazusa DNA Research Institute (Kisarazu, Japan); Primers for PCR were from Life Technologies. All of the protein sequences corresponding to the above cDNAs are listed in Table 1. Enzymes for cloning were from: NcoI, BamHI and T4 ligase (NEB, Hitchin, UK); KOD Hot Start DNA polymerase (Merck, Hertfordshire, UK); In-Fusion ${ }^{\circledR}$ HD Cloning Kit (Clontech, Takara Bio Europe SAS, SaintGermain-en-Laye, France). Bacterial cells: DH5 $\alpha$, BL21 (DE3) pLysS and SoluBL21 were a gift from Olga Mayans, University of Liverpool. The sources of other materials were as follows: LB broth and LB agar (Merck, Hertfordshire, Germany); Soniprep 150 Plus (MSE, UK); AffiGel ${ }^{\circledR}$ Heparin Gel (Bio-Rad, Hertfordshire, UK ), CM Sepharose Fast Flow, DEAE Sepharose Fast Flow, HiTrap Q HP column; empty disposable PD-10 Columns; ÄKTApurifier 100 plus (GE Healthcare, Buckinghamshire, UK).

For cell culture the following materials were used: Dulbecco's modified Eagle medium (DMEM, Life Technologies), fetal calf serum (FCS, Labtech International Ltd, East Sussex, UK), $7.5 \%$ (w/v) sodium bicarbonate (Invitrogen, Paisley, UK), $200 \mathrm{mM} \mathrm{L-glutamine} \mathrm{(Gibco),} 5 \mu \mathrm{g} / \mathrm{mL}$ insulin (Sigma-Aldrich, Dorset, UK), $5 \mu \mathrm{g} / \mathrm{mL}$ hydrocortisone (Sigma-Aldrich), bovine serum albumin (BSA; A7030, Sigma-Aldrich) for cell culture, cell culture dishes (Corning, Nottingham, UK). For SDS-PAGE and Western blotting: dried skimmed milk (Marvel, Spalding, UK), BSA (Fisher Scientific, Lougborough, UK), protease inhibitor (Roche, Burgess Hill, UK), phospho-p44/42 MAPK (T202/Y204) antibody (Cell Signalling, NEB), monoclonal $\beta$-actin antibody (Sigma-Aldrich), anti-mouse IgG, horseradish peroxidase-linked antibody (Cell Signalling, NEB), polyvinylidene fluoride (PVDF) transfer membrane (Millipore UK, Hertfordshire, UK), enhanced chemiluminescence (ECL) Western blotting reagents (GE Healthcare), Hyperfilm (GE Healthcare).

\section{DNA cloning of hexahistidine tagged FGFs (His-FGFs) and HaloTag tagged FGFs (Halo- FGFs)}

DNA encoding FGF1, FGF3, FGF6, FGF8, FGF10, FGF16, FGF17, FGF20 and FGF22 was cloned into pET-M11 such that the resulting protein would have a N-terminal 6xhis tag followed by a tobacco etch virus (TEV) cleavage site (ENLYFQ). FGF2 and FGF7 DNA sequences were previously cloned into pET-14b and pET-M11, respectively (Xu et al. 2012). 
104 A plasmid encoding Halo-FGF2 was produced by adding a HaloTag encoding DNA sequence 105 in-frame 5' to a DNA sequence encoding full-length FGF2. This construct was then used to 106 produce the other DNAs encoding Halo-FGFs (Fig 1). The plasmid pET-14b- $f g f 2$ contains NcoI 107 and BamHI cleavage sites 5' and 3' of $f g f 2$, respectively. This vector was linearized by digestion 108 with NcoI. The DNA encoding HaloTag (Fig 1: blue insert) was amplified by PCR using the 109 Halo-FGF2-Forward, AAGGAGATATACCATGCCAGAAATCGGTACTG, and Halo-FGF2110 Reverse, TCCCGGCTGCCATGGAGCTCTGAAAGTACAGATC, primers (NcoI and BamHI 111 cleavage sites underlined), and inserted into the linearized vector using In-Fusion enzyme. A 112 TEV cleavage site (Fig 1: green ellipsoid) was also included at the C-terminus of HaloTag to 113 allow release of FGF. A NotI cleavage site was also inserted 5' of the BamHI to provide an 114 additional 3' cleavage sites for cloning. The other cDNAs (FGF1, FGF3, FGF6, FGF7, FGF8, 115 FGF10, FGF16, FGF17, FGF20 and FGF22) were exchanged into the established pET-14b116 Halo-fgf2 plasmid by double-digestion with NcoI and BamHI/NotI enzymes and ligation using 117 T4 ligase (Fig 1).

\section{Protein expression and purification of His-FGFs and Halo-FGFs}

119 His-FGF7, because it is toxic like native FGF7 (Ron et al. 1993), was transformed into BL21 120 (DE3) pLysS (F- ompT hsdSB(rB-, mB-) gal dcm (DE3) pLysS (CamR)) for subsequent 121 protein expression and purification. FGF2, the other His-FGFs and Halo-FGFs were transformed 122 into SoluBL21(F-omp $\mathrm{T} h s d \mathrm{SB}(\mathrm{rB}-, \mathrm{mB}-)$ gal dcm (DE3)). The bacteria containing FGF 123 encoding plasmids were cultured at $37^{\circ} \mathrm{C}$ until the OD600 values were between 0.4 and 0.6 , and 124 then protein expression at $16^{\circ} \mathrm{C}$ was induced by adding $1 \mathrm{mM}$ isopropyl $\beta$-D-1125 thiogalactopyranoside (IPTG). The bacteria were harvested by centrifugation at $4^{\circ} \mathrm{C}, 14,000 \mathrm{~g}$ 126 for 10 minutes and the pellets frozen at $-80^{\circ} \mathrm{C}$.

127

The bacterial pellets were resuspended with the corresponding $50 \mathrm{mM}$ Tris-Cl lysate buffers ( $\mathrm{pH}$ 7.4) (Table 2), and the cells were disrupted by 5-6 cycles of sonication (30 s sonication, $60 \mathrm{~s}$ pause) on ice. Cell debris and insoluble proteins were removed by centrifugation at $4^{\circ} \mathrm{C}, 30,000$ $\mathrm{g}$ for 30 minutes. Then, the presence of soluble FGFs was tested by analysis of whole cells, the supernatant and pellet by separation of polypeptides on $12 \%(\mathrm{w} / \mathrm{v})$ SDS-PAGE and coomassie staining.

FGF2 and His-FGF7 were purified as described before (Xu et al. 2012). Soluble FGF1, FGF2, FGF3, FGF10, FGF16 and FGF17, including His-FGFs and Halo-FGFs, were loaded onto a 3 $\mathrm{mL}$ and the other soluble FGFs were loaded onto an $8 \mathrm{~mL}$ column of heparin agarose. For each FGF, different concentrations of $\mathrm{NaCl}$ (in $50 \mathrm{mM}$ Tris- $\mathrm{Cl} \mathrm{pH} \mathrm{7.4)} \mathrm{were} \mathrm{used} \mathrm{for} \mathrm{washing} \mathrm{and}$ elution (Table 2) by following the previous measurements on the electrolyte sensitivity of their heparin binding assessed by Western blot (Asada et al. 2009). The yields of His-FGFs and HaloFGFs were quantified by measuring the absorbance at $280 \mathrm{~nm}$ and the level of impurities were estimated by analysis of coomassie stained SDS-PAGE gels with ImageJ-Analyze-Gels (Ferreira \& Rasband 2012). The soluble His-FGFs eluted from heparin affinity chromatography were 
142 further purified by $\mathrm{Ni}^{2+}$ affinity chromatography. Due to the negative charge on the surface of 143 HaloTag and positive charge on the surface of FGFs, Halo-FGFs could bind to both cation- and 144 anion-exchange stationary phases. Thus, Halo-FGF1, Halo-FGF2, Halo-FGF3, Halo-FGF7 and 145 Halo-FGF10 were purified by chromatography on a $5 \mathrm{~mL}$ HiTrap Q HP column. Samples were 146 applied in $0.15 \mathrm{M} \mathrm{NaCl}$ in $\mathrm{PB}$ buffer $\left(2.7 \mathrm{mM} \mathrm{KCl}, 10 \mathrm{mM} \mathrm{Na}_{2} \mathrm{HPO}_{4}, 1.8 \mathrm{mM} \mathrm{KH}_{2} \mathrm{PO}_{4}, \mathrm{pH}\right.$ 7.4) 147 and eluted with a gradient running to $0.8 \mathrm{M} \mathrm{NaCl}$ in the same buffer. Halo-FGF6 and Halo148 FGF20 were purified by chromatography on a $3 \mathrm{~mL}$ column of CM Sepharose Fast Flow 149 followed by a $3 \mathrm{~mL}$ column of DEAE Sepharose Fast Flow. Samples were again applied in 0.15 $150 \mathrm{M} \mathrm{NaCl}$ in $\mathrm{PB}$ buffer and eluted with $0.4 \mathrm{M} \mathrm{NaCl}$ in the same buffer. The purified His-FGFs and Halo-FGFs were analysed by $12 \%(\mathrm{w} / \mathrm{v})$ SDS-PAGE followed by coomassie staining.

\section{Purification of FGFs by removing HaloTag from Halo-FGFs}

To test the accessibility of the TEV cleavage site, some Halo-FGFs, including Halo-FGF2, HaloFGF17, Halo-FGF6, Halo-FGF8 and Halo-FGF22 eluted with high concentration of $\mathrm{NaCl}$ in 50 mM Tris buffer from heparin agarose chromatography and Halo-FGF20 purified with heparin, DEAE and CM chromatography, were incubated with $2.5 \%(\mathrm{~mol} / \mathrm{mol}) \mathrm{TEV}$ protease at $4{ }^{\circ} \mathrm{C}$ overnight. In cases where the digestion products were cloudy, they were clarified by centrifugation for $30 \mathrm{~min}$ at $13,000 \mathrm{~g}, 4{ }^{\circ} \mathrm{C}$. Samples were then analysed on a $12 \%(\mathrm{w} / \mathrm{v}) \mathrm{SDS}-$ PAGE. The supernatants of the TEV digestions of Halo-FGF6 and of Halo-FGF20 were applied onto a $2 \mathrm{~mL}$ heparin agarose column, and washed as before (Table 2). FGF6 and FGF20 were eluted with $\mathrm{PB}$ buffer containing $1 \mathrm{M} \mathrm{NaCl}$ or $0.1 \mathrm{M}$ arginine and $1 \mathrm{M} \mathrm{NaCl}$, respectively. After TEV digestion, FGF17 was further purified on a $1 \mathrm{~mL}$ HiTrap SP HP cation-exchange column by washing with $0.3 \mathrm{M} \mathrm{NaCl}$ and eluting with $1 \mathrm{M} \mathrm{NaCl}$, both in $50 \mathrm{mM}$ Tris-Cl, $\mathrm{pH} 7.4$. All of the fractions from the purification steps were analysed by $12 \%(\mathrm{w} / \mathrm{v}) \mathrm{SDS}-\mathrm{PAGE}$.

\section{5}

\section{Cell culture}

Rama 27 cells were cultured in DMEM medium containing 10\% (v/v) FCS, 4 mM L-glutamine, $0.75 \%(\mathrm{w} / \mathrm{v})$ sodium bicarbonate, $50 \mathrm{ng} / \mathrm{mL}$ insulin and $50 \mathrm{ng} / \mathrm{mL}$ hydrocortisone (Rudland et al. 1984). HaCaT cells were cultured in the same medium, but without insulin and hydrocortisone (Boukamp et al. 1988). Cell number was measured with a Z1 coulter particle counter (Beckman Coulter, High Wycombe, UK).

\section{Measurement of p44/42 ${ }^{\mathrm{MAPK}}$ phosphorylation}

Cells were cultured in $3 \mathrm{~cm}$ dishes until near confluence. Then, the dishes were washed twice with phosphate-buffered saline (PBS) and $2.5 \mathrm{~mL}$ step-down medium (SDM: DMEM with 250 ng BSA, $0.75 \%(\mathrm{w} / \mathrm{v})$ sodium bicarbonate and $4 \mathrm{mM}$ L-glutamine) was added for $24 \mathrm{~h}$ (Rama 27 cells) or $48 \mathrm{~h}$ (HaCaT cells). Rama 27 and HaCaT cells were then incubated with different FGFs for $15 \mathrm{~min}$, as described in the figure legends. After the incubation, the cells were washed twice with ice-cold PBS and collected by scraping in 2X SDS-PAGE lysis buffer $(4 \%(\mathrm{w} / \mathrm{v}) \mathrm{SDS}$, $20 \%(\mathrm{v} / \mathrm{v})$ glycerol, $12 \%(\mathrm{v} / \mathrm{v})$ Tris-Cl ( $\mathrm{pH} 6.8), 2.5 \%(\mathrm{v} / \mathrm{v}) \beta$-mercaptoethanol, $0.02 \%(\mathrm{w} / \mathrm{v})$ 
179 bromophenol blue, 1 tablet of protease inhibitor and $6.8 \mathrm{~mL}$ distilled water). The cell lysates 180 were heated for 10 minutes at $98^{\circ} \mathrm{C}$ prior to SDS-PAGE.

\section{1}

\section{Western blot}

After separation by $10 \%$ SDS-PAGE, polypeptides were transferred onto a PVDF membrane. The membrane was blocked with $5 \%(\mathrm{w} / \mathrm{v})$ skimmed milk in $1 \mathrm{X}$ TBST $(50 \mathrm{mM}$ Tris-Cl, 150 $\mathrm{mM} \mathrm{NaCl}$ and $0.05 \%$ Tween-20 (v/v), pH 7.5) for 2 hours. After two washes with TBST, the membrane was incubated with phospho-p44/42 ${ }^{\mathrm{MAPK}}$ antibody (1:1000 dilution in PBST) on a shaker overnight at $4^{\circ} \mathrm{C}$. Secondary anti-mouse antibody (1:1000 dilution) was added to the membrane after three washes with PBST, 5 min each, for 1 hour at room temperature. Following three washes with PBST to remove the excess secondary antibody, the membrane was covered with $1 \mathrm{~mL}$ ECL solution and signal was detected with Hyperfilm. The same membrane was stripped with $2.5 \%(\mathrm{w} / \mathrm{v})$ SDS in PBST at $50^{\circ} \mathrm{C}$ for 1 hour and reblocked as above, before probing with $\beta$-actin antibody (1:10000 dilution). The Western blot band intensity was quantified in the same way as SDS-PAGE bands and the signal intensities of phospho$\mathrm{p} 44 / 42^{\mathrm{MAPK}}$ were normalised by dividing by the intensity of the band corresponding to $\beta$-actin and then by that of the BSA control samples.

\section{Cell growth assay}

Cell growth in Rama 27 fibroblasts was measured as before (Smith et al. 1984). Rama 27 cells were dispensed into a 24 well cell culture plates at 2000 cells/well. After $24 \mathrm{~h}$ the cells were washed twice with PBS and cultured in SDM, as described for the p44/42 ${ }^{\mathrm{MAPK}}$ phosphorylation assay for 24 hours. The SDM was then replaced and the appropriate proteins added, as described in the figure legend. After $68 \mathrm{~h}$ incubation, cells were trypsinised and the number of cells counted. 


\section{Results and Discussion}

Based on their relative expression and solubility properties, the FGFs were split into three different groups: FGFs that expressed well as soluble proteins (Group 1: FGF1, FGF2 and FGF10), FGFs that expressed at a low level (Group 2: FGF3 and FGF7), and FGFs that were insoluble when expressed in E. coli (Group 3: FGF6, FGF8, FGF16, FGF17, FGF20 and FGF22).

\section{Group 1: soluble FGFs}

After induction, bands corresponding to the expected molecular size of His-FGF1, FGF2 and His-FGF10 were apparent in the whole cell lysates (Figs 2 A, C and E, lane L, green arrow). His-FGF1 and His-FGF10 were expressed at a higher level than FGF2 in E. coli SoluBL21. After centrifugation of the cell lysates, bands corresponding to the molecular size of all three FGFs were mainly recovered in the soluble fraction (supernatant), rather than in the insoluble fraction (pellet; Figs 2 A, C, E, lanes S and P). Chromatography of the supernatants on heparin demonstrated that little of the expressed protein was present in the flow-through fraction (Figs 2 A, C, E, lane T). Weak bands corresponding to His-FGF1 and His-FGF10, but not FGF2, were observed in the wash fraction (Figs $2 \mathrm{~A}$, E, lane Wa), which may represent aggregated or less well-folded protein. The majority of the three FGFs was recovered in the high $\mathrm{NaCl}$ eluate (Figs 2 A, C and E, lane Hep), which demonstrated that these soluble FGFs bound heparin strongly. This indicated that they were likely to be properly folded, because the canonical, highest affinity heparin binding site of FGFs depends on the tertiary structure of the proteins ( $\mathrm{Xu}$ et al. 2012).

The bands corresponding to Halo-FGF1, Halo-FGF2 and Halo-FGF10 were clearly observed in the whole cell lysates and these proteins were all highly expressed in SoluBL21 cells (Figs 2 B, $\mathrm{D}$ and F, lane L, red arrow). Similarly to the His-FGF1, FGF2 and His-FGF10, after centrifugation of the whole cell lysates, the bands corresponding to the three Halo-FGFs were observed in the soluble fractions (Figs 2 B, D and F, lanes S and P). Chromatography of the soluble fractions on heparin indicated that most of Halo-FGF2 and Halo-FGF10 had bound to the column, but there was a substantial amount of Halo-FGF1 in the flow-through (Fig 2 B, D and $\mathrm{F}$, lane $\mathrm{T}$ ). This may be due to the capacity of the column for Halo-FGF1 being lower than for His-FGF1. All three Halo-FGFs were eluted from the heparin affinity column at the expected $\mathrm{NaCl}$ concentration (Figs $2 \mathrm{~B}, \mathrm{D}$ and F, lane Hep).

The yield of Halo-FGF1 and Halo-FGF10 was similar to that of the corresponding his-tagged proteins (Table 3). However, since the Halo-FGF proteins are considerably larger than the corresponding His-tagged FGF1 and FGF10, this represents a decrease in the molar amounts of FGF produced. In contrast, the yield of Halo-FGF2 was 4-fold higher (Table 3), which is only partly accounted for by the increased size of the fusion protein. The low yield of full-length FGF2 has been ascribed to the presence of secondary structure at the 5' end of the FGF2 mRNA 
241 (Knoerzer et al. 1989), and the presence of the upstream HaloTag sequence may mitigate this 242 effect.

\section{Group 2: low expression proteins}

244 The expression of His-FGF3 was weak, as was that of His-FGF7 (expressed in BL21 DE3 pLysS) 245 due to its toxicity (Ron et al. 1993)) (Figs 3A and C, lane L, S and P, green arrow). Heparin 246 chromatography of the supernatants demonstrated that the yields of soluble His-FGF3 and His247 FGF7 were quite low (Figs $3 \mathrm{~A}$ and C, lane Hep; Table 3).

248 Transformation of SoluBL21with the plasmid encoding Halo-FGF7 yielded the expected number 249 of colonies, indicating that the fusion protein was not toxic. Bands corresponding to the 250 molecular size of Halo-FGF3 and Halo-FGF7 were observed in the cell lysates (Figs 3 B and D, 251 lane L, red arrow) and in the soluble fraction obtained after centrifugation, whereas the pellet has 252 relatively weaker bands (Figs $3 \mathrm{~B}$ and D, lanes P and S), indicating that Halo-FGF3 and Halo253 FGF7 were soluble. Heparin chromatography of the soluble factions demonstrated that large 254 amounts of Halo-FGF3 and Halo-FGF7 retained their heparin binding interaction with the 255 polysaccharide (Figs $3 \mathrm{~B}$ and D, lane Hep).

256 The yields of Halo-FGF3 and of Halo-FGF7 were 21-fold and 9-fold greater than of the 257 corresponding His-tagged FGF (Table 3). Thus, the presence of the HaloTag N-terminal fusion 258 increased the amounts of FGF3 and FGF7 substantially, even after taking into account the larger 259 size of these fusion proteins (Table 3).

\section{Group 3: insoluble proteins}

261 His-FGF6, His-FGF8, His-FGF22, His-FGF17, His-FGF16 and His-FGF20 were all expressed, albeit at different levels. After centrifugation, bands corresponding to the molecular sizes of these proteins were detected in the pellet (Fig 4, compare lanes P and S, green arrow). Although small amounts of protein, such as bands corresponding to His-FGF6, His-FGF16 and His-FGF20, were observed in the supernatants (Fig. 4, lanes S), no protein were detected in the eluate from heparin chromatography, which might suggest these proteins were either small soluble aggregates or not properly folded. It has reported that FGF20 could also be solubilised by high concentrations of arginine (Maity et al. 2009), which suggests that FGF20 in the lysis buffer has a tendency to aggregate. However, arginine would compete for binding of FGFs to heparin, which reduces the utility of this approach to solubilisation.

As illustrated by SDS-PAGE, all of the bands corresponding to the molecular size of Halo-FGF6, Halo-FGF8, Halo-FGF22, Halo-FGF17, Halo-FGF16 and Halo-FGF20 were clearly observed in the whole lysates, which suggested that all six proteins expressed well in E. coli (Fig. 5, lanes L, red arrow), particularly Halo-FGF6, Halo-FGF17, Halo-FGF16 and Halo-FGF20. Although some material corresponding to the expected molecular size of these Halo-FGFs was observed in the pellet after centrifugation of the cell lysates (Fig. 5, lanes P), there were strong bands 
corresponding to Halo-FGF6, Halo-FGF16 and Halo-FGF20 and weak bands corresponding to Halo-FGF8, Halo-FGF17 and Halo-FGF22 present in the soluble fractions (Fig. 5, lanes S). Following application to a heparin affinity column, most of Halo-FGF6 in the supernatant bound to heparin and was eluted by $1 \mathrm{M} \mathrm{NaCl}$ in Tris-Cl (Fig $5 \mathrm{~A}$, lanes $\mathrm{S}$, T and Hep). Halo-FGF8 Halo-FGF17 and Halo-FGF22 also bound to the heparin-affinity column reasonably efficiently, whereas a considerable amount of Halo-FGF16 and Halo-FGF20 did not bind (Figs 5 B, C, D and $\mathrm{E}$, lanes $\mathrm{S}$ and $\mathrm{T}$ ). All four proteins could be recovered from heparin chromatography with high concentration NaCl-containing elution buffers (Table 2) (Figs 5 B, C, D and E, lane Hep). When the Halo-FGF20 in the flow-through fraction (Fig 5 F, lane T) was applied to a second heparin-affinity chromatography column, a large amount of Halo-FGF20 was found to bind and could be eluted (Fig 5 F, lane Hep2). A considerable amount of Halo-FGF16 also failed to bind to the heparin affinity column (Fig $5 \mathrm{E}$, lane T), though the bound protein was eluted with high $\mathrm{NaCl}$ (Fig $5 \mathrm{E}$, lane Hep). This suggests that the capacity of the heparin affinity column for Halo-FGF20 was exceeded. The same explanation may underlie the presence of Halo-FGF16 in the flow-through fraction, though this protein was present at a slightly lower level. However, since nothing is known about the preference of either FGF16 or FGF20 for binding structures in the polysaccharide, if these were relatively rare in heparin, the column capacity might easily be exceeded. Alternatively, the Halo-FGF16 in the flow through fraction may represent protein that is in small aggregates and/or not properly folded.

Given that the amounts of soluble His-tagged FGF6, FGF8, FGF22, FGF17, FGF16 and FGF20 were not readily detectable, it is clear that the N-terminal HaloTag fusion significantly improved the expression of soluble protein. The yield of Halo-FGF6 and Halo-FGF20 was substantial (27 $\mathrm{mg} / \mathrm{L}$ and $10 \mathrm{mg} / \mathrm{L}$, respectively, Table 3). Although a lower yield of Halo-FGF8, Halo-FGF16, Halo-FGF17 and Halo-FGF22 (1 mg/L to $2 \mathrm{mg} / \mathrm{L}$, Table 3 ) was obtained, it is nevertheless sufficient for many applications, including microscopy. However, the heparin affinity purification step did not produce entirely pure protein, as judged by coomassie staining (Figs 2, 3, 5).

\section{Further purification of some Halo-FGFs}

Four Halo-FGFs, Halo-FGF1, Halo-FGF7, Halo-FGF6 and Halo-FGF20 were chosen to determine whether the Halo-FGFs could be easily subjected to further purification, since there was clear evidence for impurities following heparin-affinity chromatography. Halo-FGF1 and Halo-FGF7 were successfully purified by Q anion-exchange chromatography (Figs 6 A and B, lane Q), which depends on the acidic isoelectric point of the HaloTag (pI: 4.77). For Halo-FGF6 and Halo-FGF20, advantage was taken of the acidic HaloTag and positive surfaces of FGFs, to enable a two-step ion-exchange purification of the eluate from heparin-affinity chromatography, using both DEAE anion and CM cation ion-exchange chromatography (Figs $6 \mathrm{C}$ and D, lane DEAE and CM). The isolated Halo-FGFs are relatively pure, as is shown on the gels (Fig. 6).

\section{Purification of FGFs by removing the HaloTag with TEV protease}


315 The inclusion of a TEV site between the sequence of the HaloTag and FGF proteins provides a 316 means to remove the HaloTag fusion partner in those instances where the HaloTag is not 317 required for analysis (or when it may interfere with such analyses). Halo-FGF2 was first 318 incubated with TEV protease to test whether the fusion protein could be cleaved by TEV. SDS319 PAGE of the TEV digestion product of Halo-FGF2 shows that almost all of the protein was 320 cleaved into the $35 \mathrm{kDa}$ HaloTag (Fig $7 \mathrm{~A}$, red arrow) and the $18 \mathrm{kDa}$ FGF2 (Fig $7 \mathrm{~A}$, green 321 arrow). Thus, the cleavage site is fully accessible to TEV protease. Both Halo-FGF17 and Halo322 FGF20 were also well digested by TEV protease and subsequently soluble FGF17 (Fig 7 B, 323 green arrow) and FGF20 (Fig 7 C, green arrow) were purified by cation-exchange and heparin

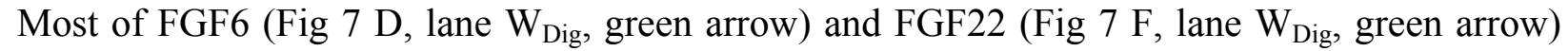
and a small proportion of FGF8 were also released from HaloTag (Figs 7 D, E and F, lane $\mathrm{W}_{\text {Dig }}$ and $\mathrm{S}$, red arrow), but these proteins were observed to aggregate upon cleavage. This suggested that these proteins were not very stable, at least in the buffer conditions used here, and required the HaloTag N-terminal fusion to remain soluble. The soluble FGF6 released by cleavage (Fig 7 $\mathrm{D}$, lane $\mathrm{S}$, green arrow) was applied to a heparin affinity column, but was observed to be concentrated at the top of the column where it formed a white aggregate. Very little protein was eluted with $1 \mathrm{M} \mathrm{NaCl}$ in PB buffer (Fig $7 \mathrm{D}$, lane E, green arrow). The disappearance of FGF8 and FGF22 in the soluble fractions after TEV digestion (Figs $7 \mathrm{E}$ and F, lane S) showed that these two proteins were also not very soluble in the present buffer conditions without the HaloTag fusion partner.

\section{Biological activities of FGFs and Halo-FGFs on Rama 27 fibroblasts and HaCaT keratinocytes}

FGF1, FGF2 and FGF6 have a preference for FGFR1c (Zhang et al. 2006a), the predominant receptor expressed by Rama 27 fibroblasts (Delehedde et al. 2000; Zhu et al. 2010). When Rama 27 cells were stimulated with $25 \mathrm{pM}$ FGF2 for $15 \mathrm{~min}$, strong bands corresponding to dually phosphorylated p44/42 ${ }^{\mathrm{MAPK}}$ were apparent (Fig. $8 \mathrm{~A}$ ), as observed previously (Delehedde et al. 2000; Zhu et al. 2010). Halo-FGF2 caused a similar stimulation of phosphorylation of p44/42 $2^{\mathrm{MAPK}}$ (Fig. 8 A). In contrast, the $25 \mathrm{pM}$ or $50 \mathrm{pM}$ HaloTag protein alone did not appreciably stimulate $\mathrm{p} 44 / 42^{\mathrm{MAPK}}$ phosphorylation. Therefore, the activity of Halo-FGF2 in this assay is equivalent to that of FGF2 (Fig 9). In the case of FGF1, the N-terminal HaloTag also did not affect the ability of the growth factor to stimulate the phosphorylation of p44/42 MAPK (Fig. 8 A). FGF6 is not soluble without the HaloTag, so only the activity of the fusion protein could be tested, and it was found to stimulate the phosphorylation of p44/42 $2^{\mathrm{MAPK}}$ to an extent similar to that observed with FGF1 and FGF2 (Fig. 8 A). Since FGF6 has the same receptor preference as FGF1 and FGF2 (Zhang et al. 2006a), this suggests Halo-FGF6 was fully active.

FGF8, FGF16, FGF17 and FGF20 have a preference for FGFR3c, but they are also able to activate FGFR1c, though higher concentrations of growth factor are required to elicit activity 
(Zhang et al. 2006a). When 500 pM HaloTag was added to the cells, there was no detectable increase in phosphorylation of p44/42 ${ }^{\mathrm{MAPK}}$, whereas Halo-FGF8, Halo-FGF20 and FGF20 at concentrations comparable to those used in previous work (Zhang et al. 2006a) were all found to stimulate the phosphorylation of p44/42 MAPK (Fig. 8 B). In contrast, Halo-FGF16, Halo-FGF17 and FGF17 did not cause a detectable increase in phosphorylation of p44/42 ${ }^{\text {MAPK }}$ (Fig. 9). These data indicate that Halo-FGF8, FGF20 and Halo-FGF20 have biological activities on Rama 27 fibroblasts. The absence of stimulation of phosphorylation of p44/42 ${ }^{\mathrm{MAPK}}$ by Halo-FGF16 may reflect the fact that the ability of this FGF to activate FGFR1c is considerably lower than that of FGF8, FGF17 and FGF20 (Zhang et al. 2006a). However, the absence of stimulation of phosphorylation of p44/42 $\mathrm{MAPK}$ by FGF17 and Halo-FGF17 is more puzzling. One explanation may be that FGF16, and perhaps FGF17, do not cause the FGFR to activate strongly early biochemical signals that converge on $\mathrm{p} 44 / 42^{\mathrm{MAPK}}$. To test this, the capacity of Halo-FGF16, Halo-FGF17 and FGF17 to stimulate cell growth was measured in Rama 27 fibroblasts. The results show that $10 \mathrm{nM}$ HaloTag only weakly stimulated the growth of Rama27 fibroblasts. Halo-FGF16 caused the number of cells to double compared to the negative control, and this level was significantly ( $p=0.015$, Tukey test, OriginPro 9) above that observed in the presence of HaloTag alone (Fig. 8 C). Halo-FGF17 and FGF17 were even more effective, as they caused a 3to 4 -fold increase in the number of cells (Fig. 8 C). These results demonstrated that Halo-FGF16, FGF17 and Halo-FGF17 possess biological activities of similar potency as observed by others in growth assays (Zhang et al. 2006a).

The activity of members of the FGF7 subfamily were tested on HaCaT keratinocytes, as this cell type expresses the cognate receptor for these FGFs, FGFR2b (Ron et al. 1993). HaCaT cells have previously been shown to express more $\mathrm{p} 42^{\mathrm{MAPk}}$ than $\mathrm{p} 44^{\mathrm{MAPk}}$ (Delehedde et al. 2002). The data show clearly that HaloTag alone did not stimulate the phosphorylation of p44/42MAPK (Fig. 8 D). In contrast, FGF3, FGF7 and FGF10, and the corresponding HaloTag fusion proteins stimulated p44/42 $\mathrm{MAPK}$ phosphorylation (Fig. $8 \mathrm{C}$ ). FGF22, which is only soluble as a HaloTag fusion protein, also stimulated $\mathrm{p} 44 / 42^{\mathrm{MAPK}}$ phosphorylation to an extent similar to that seen with the other members of the subfamily (Fig. 8 D). Thus, these Halo-FGFs retain full biological activity in this assay.

\section{Conclusion}

In this study, we identified four useful properties of N-terminal HaloTag fusions for the production of biologically active FGFs: i) using the HaloTag can increase the yield of low expression FGFs, ii) the HaloTag rendered FGF7 non-toxic; iii) for the insoluble FGFs, the HaloTag enabled E.coli to express more soluble protein at low induction temperatures and maintain solubility during isolation and storage; iv) a consequence of the low isoelectric point of HaloTag was that anion-exchange chromatography could be used as an orthogonal step in the purification of the Halo-FGFs. However, there are clearly limitations, for example, some of the 
391 FGFs did not retain solubility following cleavage from the HaloTag. This may reflect the fact 392 that no single solubilisation tag is a universal panacea for resolving the problems of protein 393 expression (Costa et al. 2014). Nevertheless, because the HaloTag can enhance expression of 394 soluble protein and provide a means to label FGF protein with different fluorescent dyes and 395 quantum dots, e.g., (Los et al. 2008; Zhang et al. 2006b) it is clearly a versatile and useful tool 396 for these two purposes and, therefore, worthwhile exploring as a part of experimental strategy 397 with these aims. 


\section{PeerJ Reviewing Manuscript}

402

403

404

405

406

407

408

409

410

411

412

413

414

415

416

417

418

419

420

421

422

423

424

425

426

427

428

429

430

431

432

433

434

435

436

437

438

439

440

441

442

443

444

\section{Acknowledgements}

Xianqing Mao would like to thank Monika Dieterle for help with cloning Halo-FGF3.

\section{References}

Asada M, Shinomiya M, Suzuki M, Honda E, Sugimoto R, Ikekita M, and Imamura T. 2009. Glycosaminoglycan affinity of the complete fibroblast growth factor family. Biochim Biophys Acta 1790:40-48.

Beenken A, and Mohammadi M. 2009. The FGF family: biology, pathophysiology and therapy. Nature Reviews Drug Discovery 8:235-253.

Boukamp P, Petrussevska RT, Breitkreutz D, Hornung J, Markham A, and Fusenig NE. 1988. Normal keratinization in a spontaneously immortalized aneuploid human keratinocyte cell line. Journal of Cell Biology 106:761-771.

Costa S, Almeida A, Castro A, and Domingues L. 2014. Fusion tags for protein solubility, purification, and immunogenicity in Escherichia coli: the novel Fh8 system. Frontiers in Microbiology 5.

Danilenko DM, Montestruque S, Philo JS, Li TS, Hill D, Speakman J, Bahru M, Zhang MS, Konishi O, Itoh N, Chirica M, Delaney J, Hernday N, Martin F, Hara S, Talvenheimo J, Narhi LO, and Arakawa T. 1999. Recombinant rat fibroblast growth factor-16: Structure and biological activity. Archives of Biochemistry and Biophysics 361:34-46.

Delehedde M, Lyon M, Vidyasagar R, McDonnell TJ, and Fernig DG. 2002. Hepatocyte growth factor/scatter factor binds to small heparin-derived oligosaccharides and stimulates the proliferation of human HaCaT keratinocytes. Journal of Biological Chemistry 277:12456-12462.

Delehedde M, Seve M, Sergeant N, Wartelle I, Lyon M, Rudland PS, and Fernig DG. 2000. Fibroblast growth factor-2 stimulation of $\mathrm{p} 42 / 44$ (MAPK) phosphorylation and I kappa B degradation is regulated by heparan sulfate/heparin in rat mammary fibroblasts. Journal of Biological Chemistry 275:33905-33910.

Duchesne L, Gentili D, Comes-Franchini M, and Fernig DG. 2008. Robust ligand shells for biological applications of gold nanoparticles. Langmuir 24:13572-13580.

Duchesne L, Octeau V, Bearon RN, Beckett A, Prior IA, Lounis B, and Fernig DG. 2012. Transport of fibroblast growth factor 2 in the pericellular matrix is controlled by the spatial distribution of its binding sites in heparan sulfate. PLOS Biology 10:e1001976.

Ferreira T, and Rasband W. 2012. ImageJ user guide - Analyze - Gels. Available at http://rsbweb.nih.gov/ij/docs/guide/146-30.html\#toc-Subsection-30.13 (accessed 09 December 2014).

Huang ZH, Hwang P, Watson DS, Cao LM, and Szoka FC. 2009. Tris-nitrilotriacetic acids of subnanomolar affinity toward hexahistidine tagged molecules. Bioconjugate Chemistry 20:1667-1672.

Itoh N. 2007. The FGF families in humans, mice, and zebrafish: Their evolutional processes and roles in development, metabolism, and disease. Biological \& Pharmaceutical Bulletin 30:1819-1825.

Jeffers M, McDonald WF, Chillakuru RA, Yang MJ, Nakase H, Deegler LL, Sylander ED, Rittman B, Bendele A, Sartor RB, and Lichenstein HS. 2002. A novel human fibroblast growth factor treats experimental intestinal inflammation. Gastroenterology 123:1151-1162.

Kalinina J, Byron SA, Makarenkova HP, Olsen SK, Eliseenkova AV, Larochelle WJ, Dhanabal M, Blais S, Ornitz DM, Day LA, Neubert TA, Pollock PM, and Mohammadi M. 2009. Homodimerization controls the fibroblast growth factor 9 subfamily's receptor binding and heparan sulfatedependent diffusion in the extracellular matrix. Molecular and Cellular Biology 29:4663-4678.

Ke YQ, Fernig DG, Smith JA, Wilkinson MC, Anandappa SY, Rudland PS, and Barraclough R. 1990. High level production of human acidic fibroblast growth factor in Escherichia coli cells inhibition of 
DNA synthesis in Rat mammary fibroblasts at high concentrations of growth factor. Biochemical and Biophysical Research Communications 171:963-971.

Knoerzer W, Binder HP, Schneider K, Gruss P, Mccarthy JEG, and Risau W. 1989. Expression of synthetic genes encoding bovine and human basic fibroblast growth-factors (bFGFs) in escherichia-coli. Gene 75:21-30.

Lata S, Reichel A, Brock R, Tampe R, and Piehler J. 2005. High-affinity adaptors for switchable recognition of histidine-tagged proteins. Journal of the American Chemical Society 127:1020510215.

Lin XH. 2004. Functions of heparan sulfate proteoglycans in cell signaling during development. Development 131:6009-6021.

Loo BM, and Salmivirta M. 2002. Heparin/heparan sulfate domains in binding and signaling of fibroblast growth factor 8b. Journal of Biological Chemistry 277:32616-32623.

Los GV, Encell LP, McDougall MG, Hartzell DD, Karassina N, Zimprich C, Wood MG, Learish R, Ohane RF, Urh M, Simpson D, Mendez J, Zimmerman K, Otto P, Vidugiris G, Zhu J, Darzins A, Klaubert DH, Bulleit RF, and Wood KV. 2008. HatoTag: A novel protein labeling technology for cell imaging and protein analysis. ACS Chemical Biology 3:373-382.

Macarthur CA, Lawshe A, Xu JS, Santosocampo S, Heikinheimo M, Chellaiah AT, and Ornitz DM. 1995. Fgf-8 isoforms activate receptor splice forms that are expressed in mesenchymal regions of mouse development. Development 121:3603-3613.

Maity H, Karkaria C, and Davagnino J. 2009. Effects of $\mathrm{pH}$ and arginine on the solubility and stability of a therapeutic protein (fibroblast growth factor 20): relationship between solubility and stability. Current Pharmaceutical Biotechnology 10:609-625.

Ohana RF, Encell LP, Zhao K, Simpson D, Slater MR, Urh M, and Wood KV. 2009. HaloTag7: A genetically engineered tag that enhances bacterial expression of soluble proteins and improves protein purification. Protein Expression and Purification 68:110-120.

Ornitz DM. 2000. FGFs, heparan sulfate and FGFRs: complex interactions essential for development. Bioessays 22:108-112.

Pizette S, Batoz M, Prats H, Birnbaum D, and Coulier F. 1991. Production and functional-characterization of human recombinant FGF-6 protein. Cell Growth and Differentiation 2:561-566.

Ron D, Bottaro DP, Finch PW, Morris D, Rubin JS, and Aaronson SA. 1993. Expression of biologicallyactive recombinant keratinocyte growth-factor - structure-function analysis of amino-terminal truncation mutants. Journal of Biological Chemistry 268:2984-2988.

Roullier V, Clarke S, You C, Pinaud F, Gouzer G, Schaible D, Marchi-Artzner V, Piehler J, and Dahan M. 2009. High-affinity labeling and tracking of individual histidine-tagged proteins in live cells using $\mathrm{Ni}^{2+}$ Tris-nitrilotriacetic acid quantum dot conjugates. Nano Letters 9:1228-1234.

Rudland PS, Twiston Davies AC, and Tsao SW. 1984. Rat mammary preadipocytes in culture produce a trophic agent for mammary epithelia-prostaglandin E2. J Cell Physiol 120:364-376.

Smith JA, Winslow DP, and Rudland PS. 1984. Different growth factors stimulate cell division of rat mammary epithelial, myoepithelial and stromal cell lines in culture. J Cell Physiol 119:120-126.

Susumu K, Medintz IL, Delehanty JB, Boeneman K, and Mattoussi H. 2010. Modification of poly(ethylene glycol)-capped quantum dots with nickel nitrilotriacetic acid and self-assembly with histidinetagged proteins. Journal of Physical Chemistry C 114:13526-13531.

Tinazli A, Tang JL, Valiokas R, Picuric S, Lata S, Piehler J, Liedberg B, and Tampe R. 2005. High-affinity chelator thiols for switchable and oriented immobilization of histidine-tagged proteins: $A$ generic platform for protein chip technologies. Chemistry-a European Journal 11:5249-5259. Turner N, and Grose R. 2010. Fibroblast growth factor signalling: from development to cancer. Nature Reviews Cancer 10:116-129. 
Uchinomiya S, Nonaka H, Fujishima S, Tsukiji S, Ojida A, and Hamachi I. 2009. Site-specific covalent labeling of His-tag fused proteins with a reactive Ni(II)-NTA probe. Chemical Communications 39:5880-5882.

Vogel A, Rodriguez C, and IzpisuaBelmonte JC. 1996. Involvement of FGF-8 in initiation, outgrowth and patterning of the vertebrate limb. Development 122:1737-1750.

Xu RY, Ori A, Rudd TR, Uniewicz KA, Ahmed YA, Guimond SE, Skidmore MA, Siligardi G, Yates EA, and Fernig DG. 2012. Diversification of the structural determinants of fibroblast growth factorheparin interactions implications for binding specificity. Journal of Biological Chemistry 287:40061-40073.

Yu S, Burkhardt M, Nowak M, Ries J, Petrásek Z, Scholpp S, Schwille P, and Brand M. 2009. FGF8 morphogen gradient is formed by a source-sink mechanism with freely diffusing molecules. Nature 461:533-536.

Zhang XQ, Ibrahimi OA, Olsen SK, Umemori H, Mohammadi M, and Ornitz DM. 2006a. Receptor specificity of the fibroblast growth factor family - The complete mammalian FGF family. Journal of Biological Chemistry 281:15694-15700.

Zhang Y, So MK, Loening AM, Yao HQ, Gambhir SS, and Rao JH. 2006b. HaloTag protein-mediated sitespecific conjugation of bioluminescent proteins to quantum dots. Angewandte ChemieInternational Edition 45:4936-4940.

Zhu HY, Duchesne L, Rudland PS, and Fernig DG. 2010. The heparan sulfate co-receptor and the concentration of fibroblast growth factor- 2 independently elicit different signalling patterns from the fibroblast growth factor receptor. Cell Communication and Signaling 8:14. 
1

Cloning strategy for plasmids encoding Halo-FGFs

DNA encoding HaloTag was inserted $5^{\prime}$ of the FGF2 coding sequence with the In-Fusion HD enzyme. Subsequently, a Notl cleavage site was added 5' to the BamHI site and other FGFs were exchanged into the plasmid using the digestion-ligation cloning method. A cartoon structure of Halo-FGF is presented in the middle of this figure.

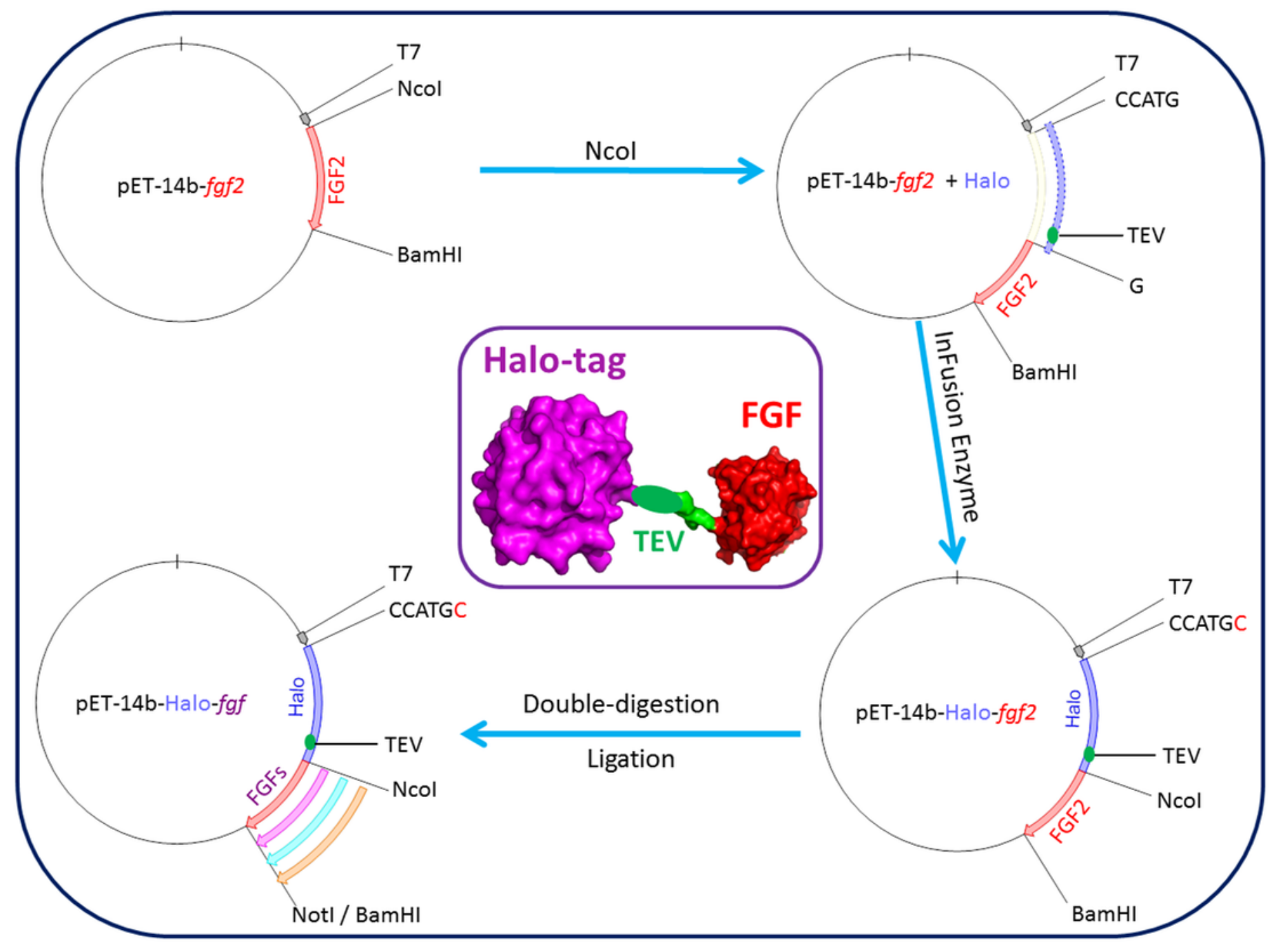


2

Expression and heparin affinity purification of His-FGF1, FGF2, His-FGF10, Halo-FGF1, Halo-FGF2 and Halo-FGF10

Following induction of expression with IPTG, cells were lysed by sonication and the insoluble material collected by centrifugation. The supernatant was subjected to heparin-afinity chromatography and samples were then analysed by SDS-PAGE and coomassie staining. Lane M, markers; L, sonicated whole cell lysate; P, pellet following centrifugation of lysate; S, corresponding supernatant; $T$, unbound, flow-through fraction from heparin-affinity chromatography; Wa, wash of heparin-affinity column (Table 2); Hep, high $\mathrm{NaCl}$ eluate of heparin-affinity column (Table 2). Green arrows: FGF or His-FGF; red arrows: Halo-FGF. 

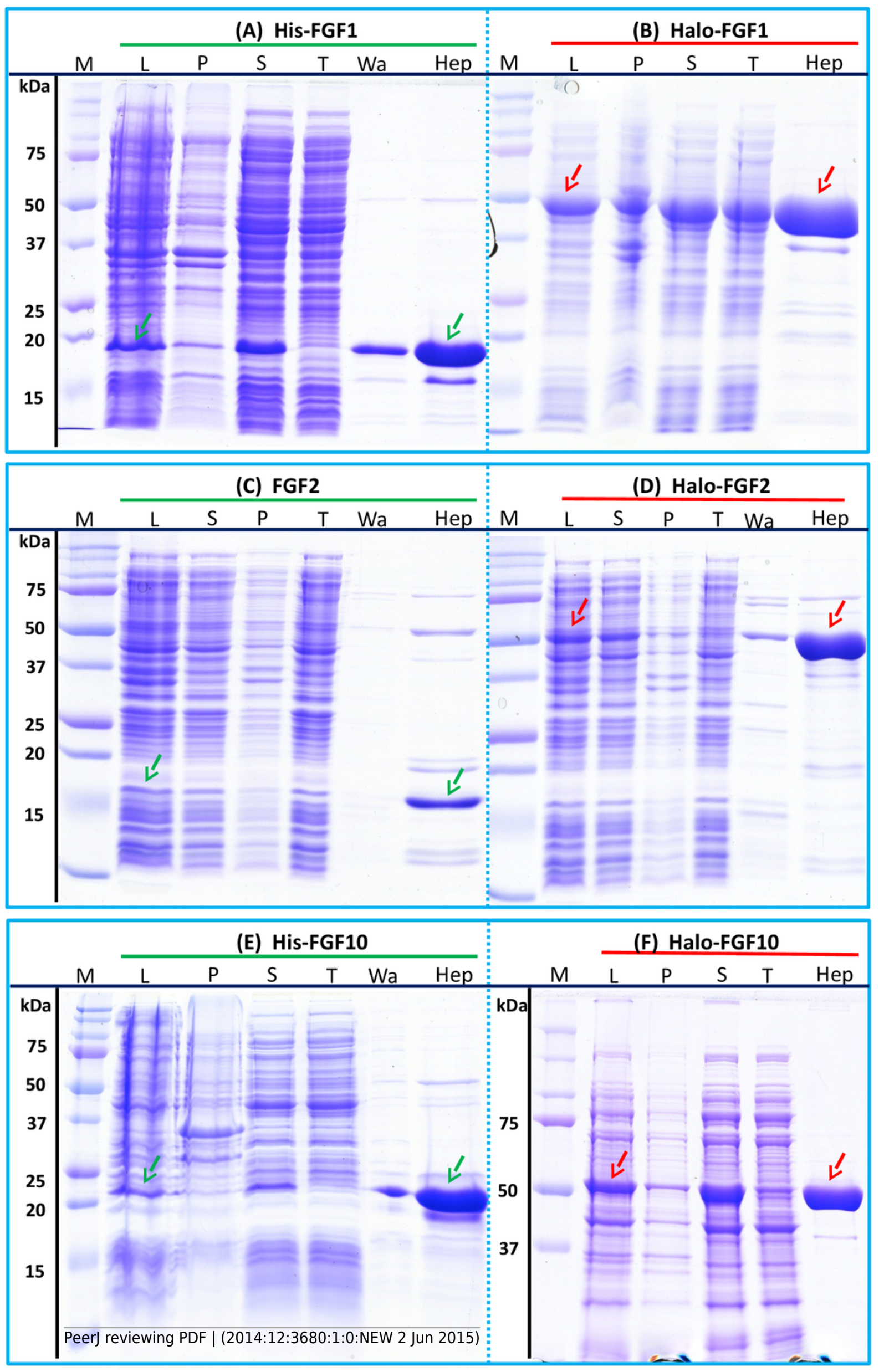


\section{3}

Expression and heparin binding-affinity chromatography of His-FGF3, His-FGF7, HaloFGF3 and Halo-FGF7

Following induction of expression with IPTG, cells were lysed by sonication and the insoluble material collected by centrifugation. The supernatant was subjected to heparin-afinity chromatography and samples were then analysed by SDS-PAGE and coomassie staining. Lane M, markers; L, sonicated whole cell lysate; P, pellet following centrifugation of lysate; S, corresponding supernatant; $T$, unbound, flow-through fraction from heparin-affinity chromatography; Hep, high [ $\mathrm{NaCl}$ eluate of heparin-affinity column (Table 2). Green arrows: His-FGF; red arrows: Halo-FGF. 


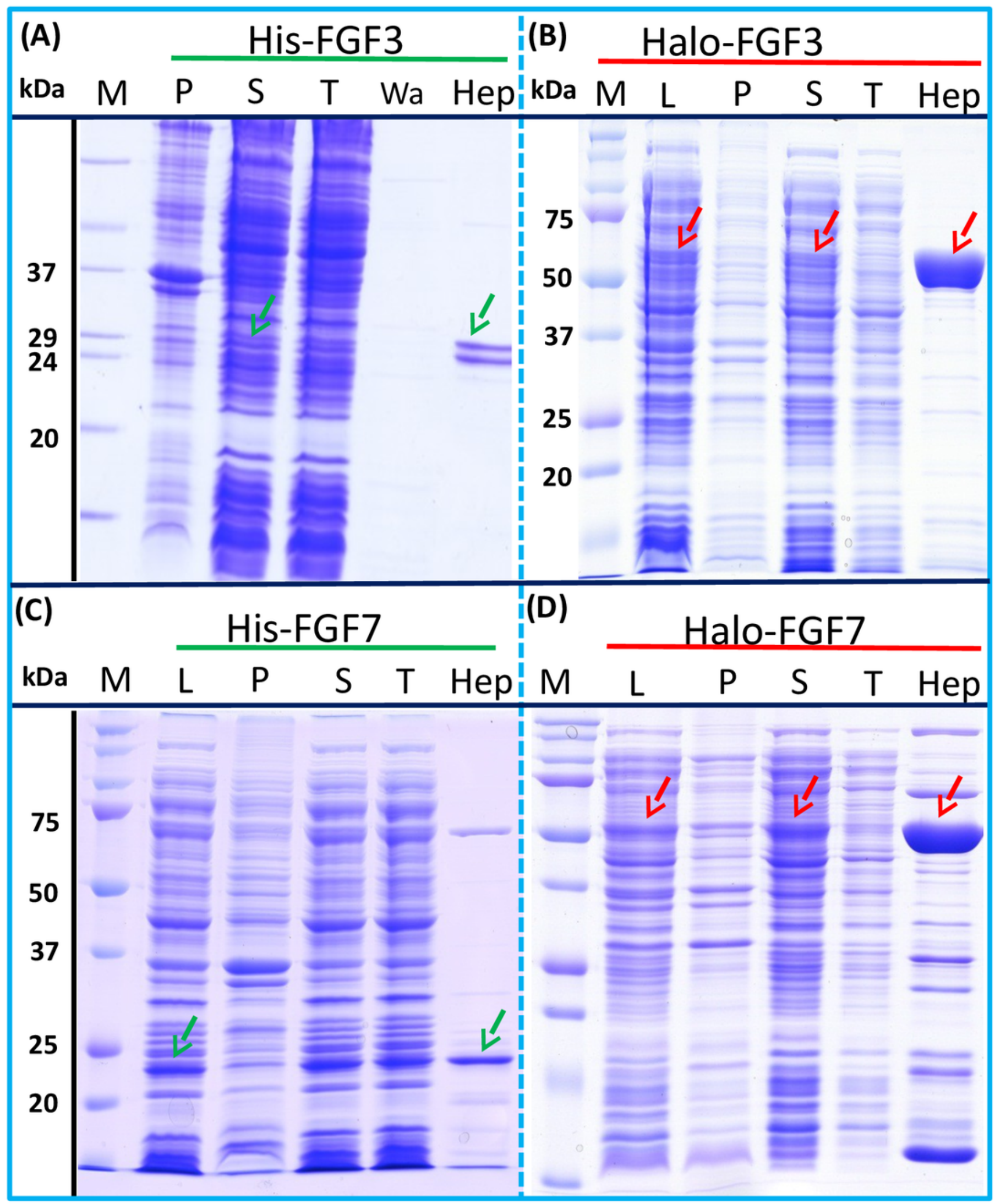




\section{4}

Expression test of His-FGF6, His-FGF8, His-FGF22, His-FGF17, His-FGF16 and His-FGF20

Following induction of expression with IPTG, cells were lysed by sonication and the insoluble material collected by centrifugation. The whole cell lysate, supernatant and pellet were analysed by SDS-PAGE and coomassie staining. Lane M, markers; L, sonicated whole cell lysate; $P$, pellet following centrifugation of lysate; $S$, corresponding supernatant. Green arrows: His-FGF. 
PeerJ Reviewing Manuscript

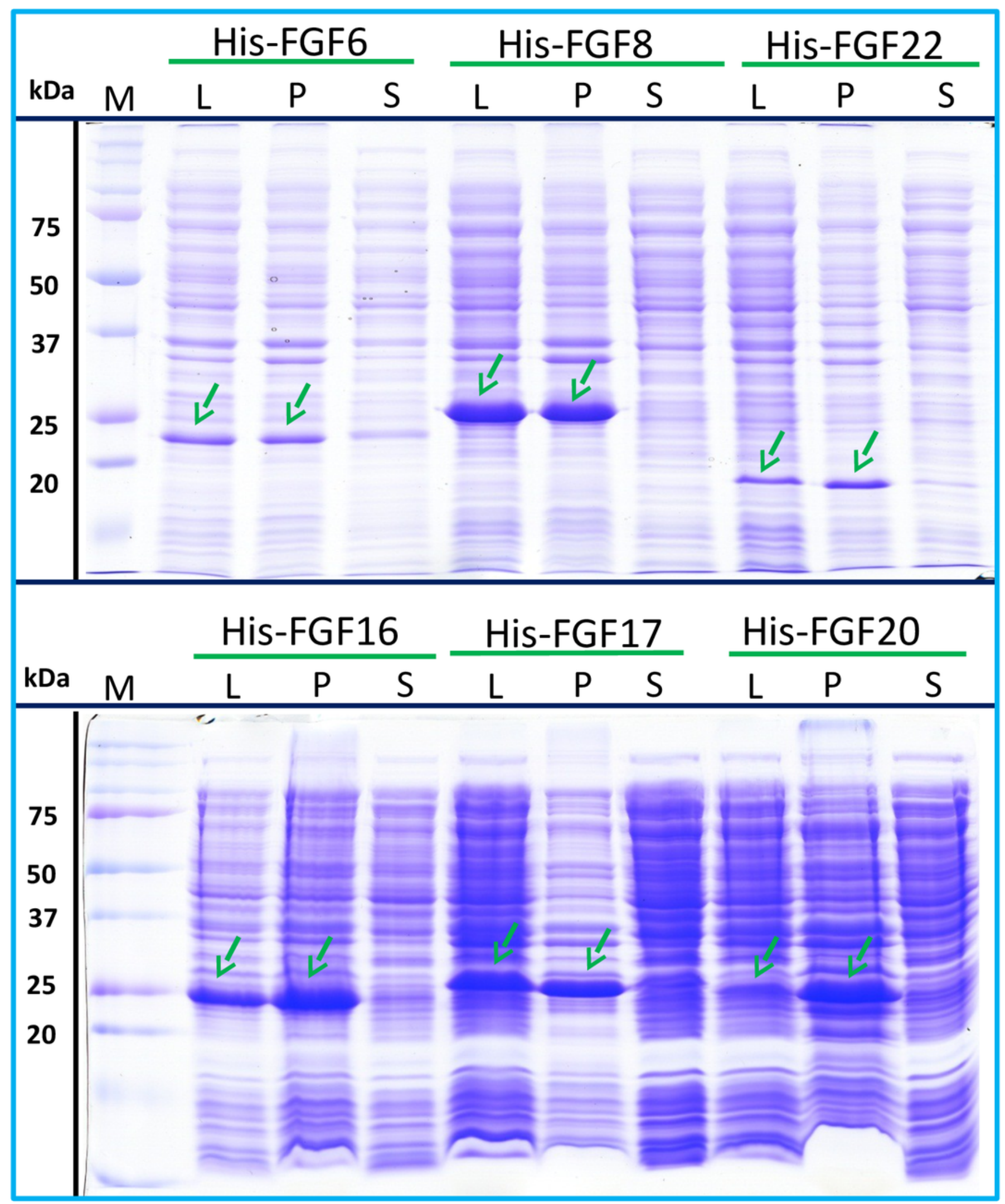




\section{5}

Expression and heparin affinity purification of Halo-FGF6, Halo-FGF8, Halo-FGF22, HaloFGF17, Halo-FGF16 and Halo-FGF20

Following induction of expression with IPTG, cells were lysed by sonication and the insoluble material collected by centrifugation. The supernatant was subjected to heparin-afinity chromatography and samples were then analysed by SDS-PAGE and coomassie staining. Lane M, markers; L, sonicated whole cell lysate; P, pellet following centrifugation of lysate; S, corresponding supernatant; $\mathrm{T}$, unbound, flow-through fraction from heparin-affinity chromatography; Hep, high $\mathrm{NaCl}$ eluate of heparin-affinity column (Table 2). Red arrows: Halo-FGF. 


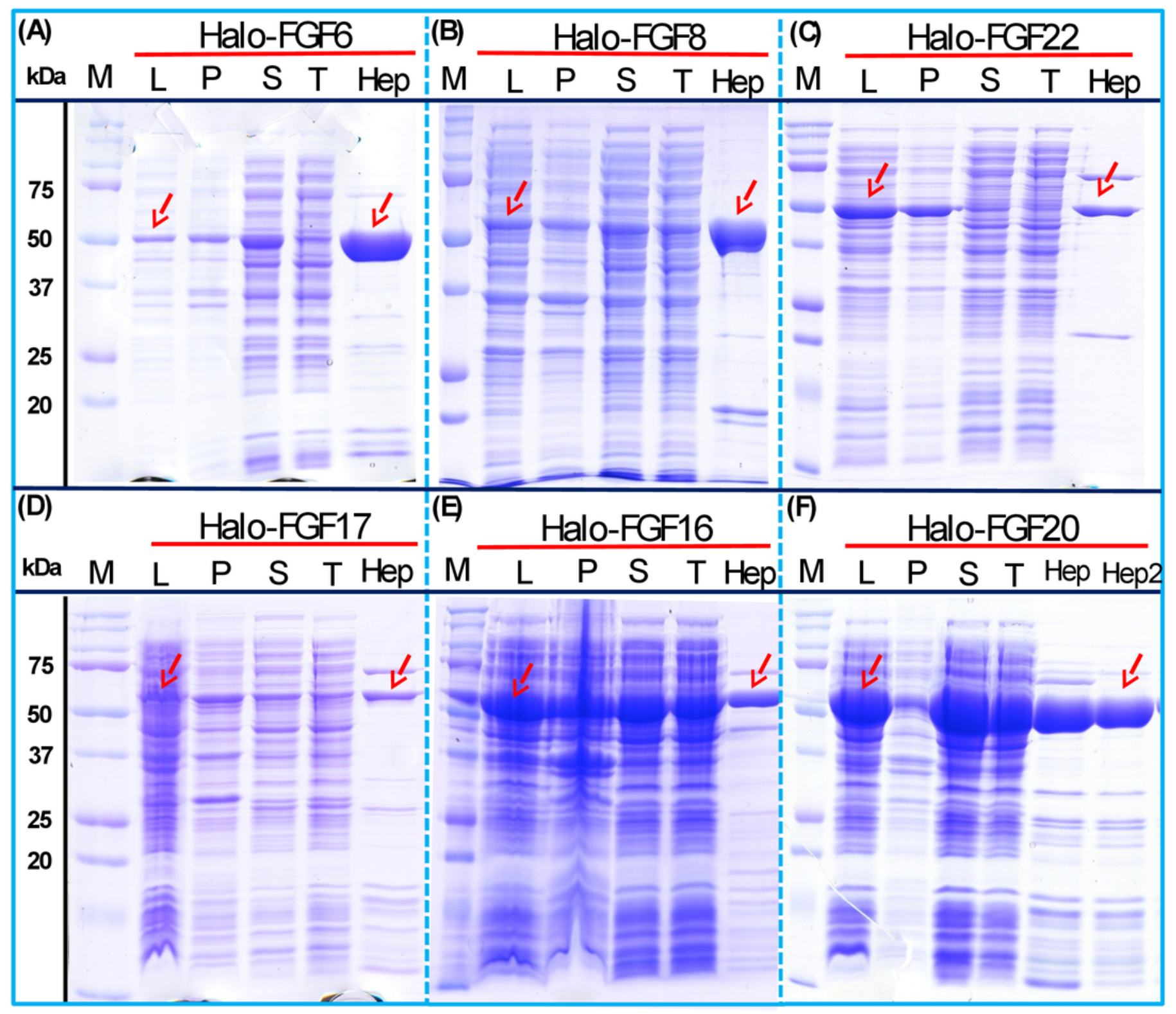




\section{6}

Further purification of the heparin affinity eluate of Halo-FGF1, Halo-FGF6, Halo-FGF7 and Halo-FGF20 by ion-exchange chromatography

The soluble Halo-FGF1 and Halo-FGF7 eluted from heparin chromatography was purified using $\mathrm{Q}$ ion-exchange chromatography, while CM and DEAE ion-exchange chromatography were used to purify Halo-FGF6 and Halo-FGF20. Lane M, markers; Hep, eluate from heparin chromatography as is shown above; $T$, unbound, flow-through fraction from ion-exchange chromatography; Q, peak fractions collected from Q HP chromatography; DEAE eluate from DEAE chromatography, two identical samples; CM, eluate from CM chromatography. Red arrows: Halo-FGF. 


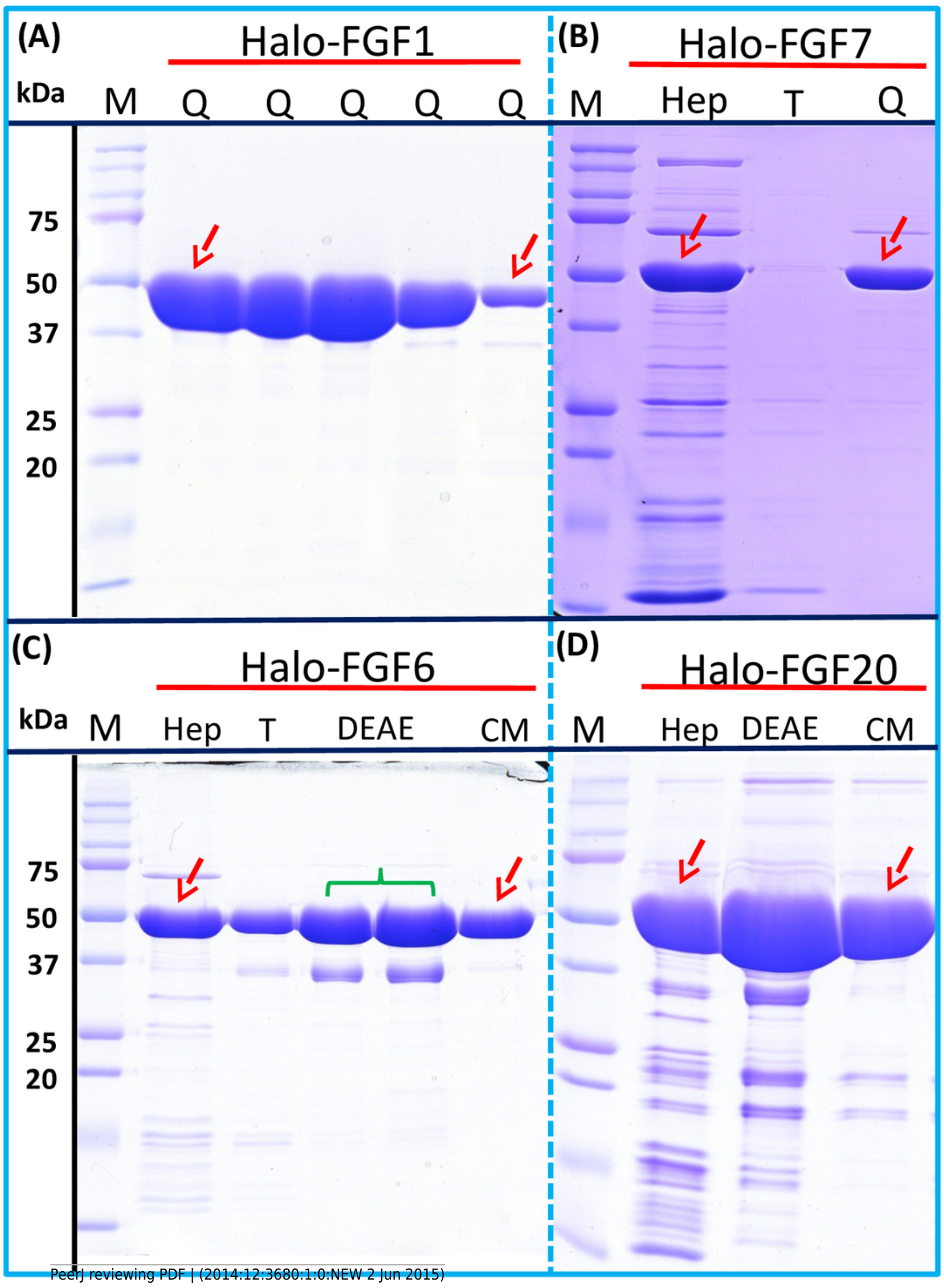




\section{7}

Cleavage of Halo-FGFs by TEV and purification

The eluates of Halo-FGF2, Halo-FGF17, Halo-FGF6, Halo-FGF8 and Halo-FGF22 from heparinaffinity chromatography and the Halo-FGF20 purified by heparin and ion-exchange chromatography were digested by TEV protease to separate HaloTag and FGF. Halo-FGF6, Halo-FGF8 and Halo-FGF22 became turbid after digestion and these samples were clarified by centrifugation. Then, the samples containing FGF6 and FGF20 were subjected to heparin chromatography and that of FGF17 to SP HP cation-exchange chromatography. Lanes M, markers; Hep, eluate from heparin chromatography; $W_{D i g}$, whole digestion product of HaloFGFs purified by heparin chromatography; $T$, unbound, flow-through fraction from heparin chromatography; Wa, wash of SP HP cation-exchange chromatography; P, pellet following centrifugation of product of TEV digestion; $\mathrm{S}$, supernatant after the centrifugation; $\mathrm{E}$, high $\mathrm{NaCl}$ eluate of heparin or SP cation-exchange chromatography. Green arrows: FGF; red arrows: HaloTag. 


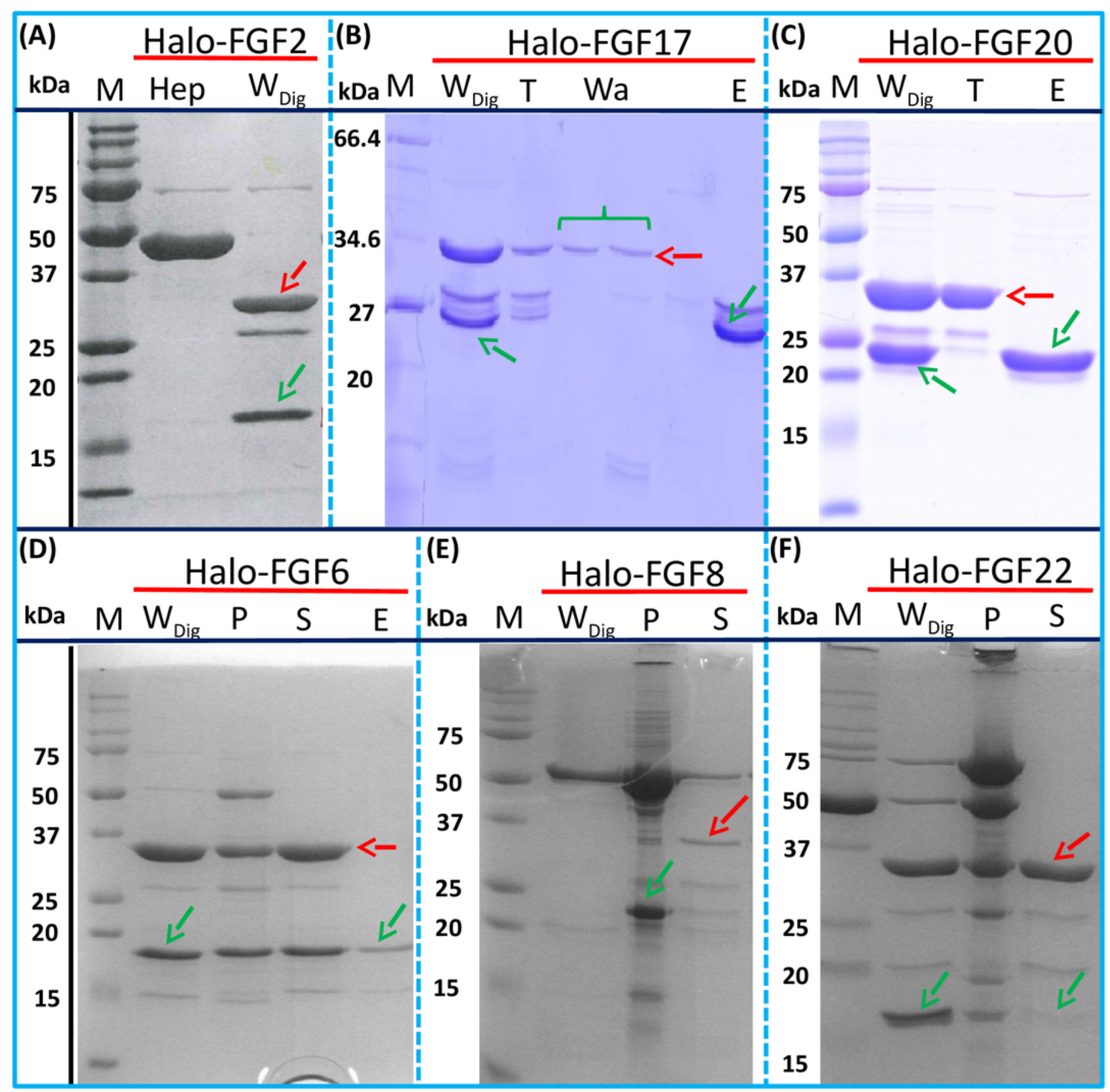


8

Activities of FGFs on Rama 27 fibroblasts and HaCaT keratinocytes

Cells were grown in $3 \mathrm{~cm}$ diameter dishes (Western blots) or 24-well plates growth assay, as described in the Materials and Methods. After incubation in SDM for $24 \mathrm{~h}$ (Rama 27) or $48 \mathrm{~h}$ (HaCaT), cells were stimulated with the FGF protein for $15 \mathrm{~min}$ (Western blot) or $68 \mathrm{~h}$ (cell growth assay). (A) Stimulation of p44/42 $2^{\text {MAPK }}$ phosphorylation by 25 pM HaloTag, FGF2, HaloFGF2 and 50 pM HaloTag, FGF1, Halo-FGF1 and Halo-FGF6 in Rama 27 fibroblasts. (B) Stimulation of p44/42 ${ }^{\text {MAPK }}$ phosphorylation by 500 pM HaloTag, Halo-FGF8, Halo-FGF16, FGF17, Halo-FGF17, FGF20 and Halo-FGF20 in Rama 27 fibroblasts. (C) Stimulation of cell growth of Rama 27 fibroblasts by $10 \mathrm{nM}$ Halo-FGF16, FGF17 and Halo-FGF17. (D) Stimulation of p44/42 ${ }^{\text {MAPK }}$ phosphorylation by 300 pM His-FGF3, Halo-FGF3 and Halo-FGF22, 30 pM HisFGF7, Halo-FGF7, His-FGF10 and Halo-FGF10 in HaCaT cells. 


\begin{tabular}{|c|c|c|c|c|c|c|c|c|c|c|c|}
\hline \multicolumn{12}{|c|}{ (A) } \\
\hline HaloTag & - & 25 & 50 & - & - & - & - & - & - & 25 & 50 \\
\hline FGF1 & - & - & - & + & - & - & - & - & - & - & - \\
\hline Halo-FGF1 & - & - & - & - & + & - & - & - & - & - & - \\
\hline FGF2 & - & - & - & - & - & + & - & - & - & - & - \\
\hline Halo-FGF2 & - & - & - & - & - & - & + & - & - & - & - \\
\hline Halo-FGF 6 & - & - & - & - & - & - & - & + & - & - & - \\
\hline P-p44/42 & & $=$ & $2=$ & & $\approx$ & $=$ & & $=$ & $\bar{E}$ & $\overline{-}$ & $\square$ \\
\hline$\beta$-Actin & & & & & & & & & & & \\
\hline
\end{tabular}

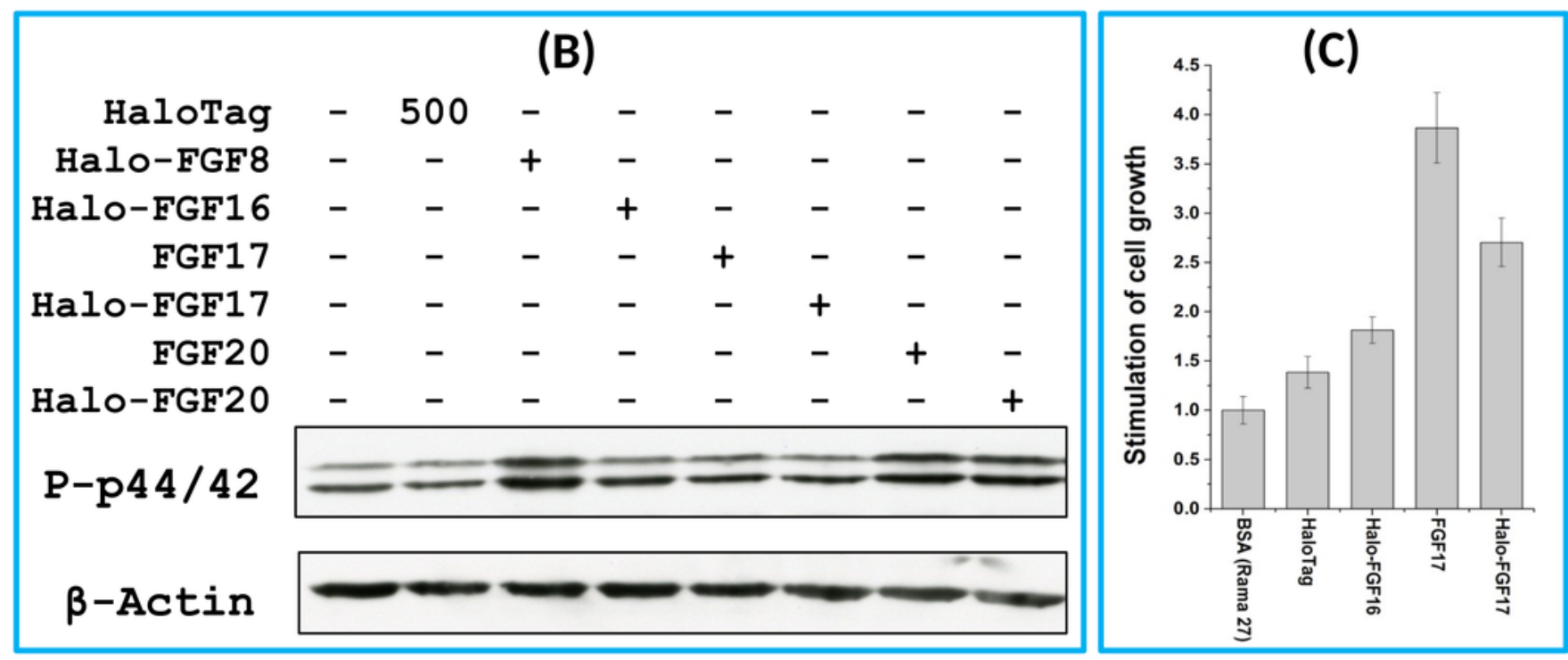

\begin{tabular}{|c|c|c|c|c|c|c|c|c|c|c|c|c|}
\hline \multicolumn{13}{|c|}{ (D) } \\
\hline HaloTag & - & - & - & - & - & - & - & - & - & 30 & 100 & 300 \\
\hline FGF3 & - & + & - & - & - & - & - & - & - & - & - & - \\
\hline Halo-FGF3 & - & - & + & - & - & - & - & - & - & - & - & - \\
\hline FGF7 & - & - & - & + & - & - & - & - & - & - & - & - \\
\hline Halo-FGF7 & - & - & - & - & + & - & - & - & - & - & - & - \\
\hline FGF10 & - & - & - & - & - & + & - & - & - & - & - & - \\
\hline Halo-FGF10 & - & - & - & - & - & - & + & - & - & - & - & - \\
\hline Halo-FGF22 & - & - & - & - & - & - & - & + & - & - & - & - \\
\hline $\mathrm{P}-\mathrm{p} 44 / 42$ & 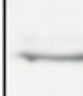 & $=$ & $=$ & $=$ & - & $=$ & $=$ & $\Longrightarrow$ & & $=$ & $=$ & - \\
\hline$\beta$-Actin & $=$ & $=$ & - & $=$ & & $=$ & $=$ & $=$ & & & 15 & $=$ \\
\hline
\end{tabular}


9

Quantification of p44/42 MAPK phosphorylation

The band intensities from two experiments were quantified with imageJ and normalised to the BSA control to compare the similarities and differences of stimulation of phosphorylation p44/42 ${ }^{\text {MPR }}$ by different FGFs. Results are the mean with the actual values from two independent experiments.

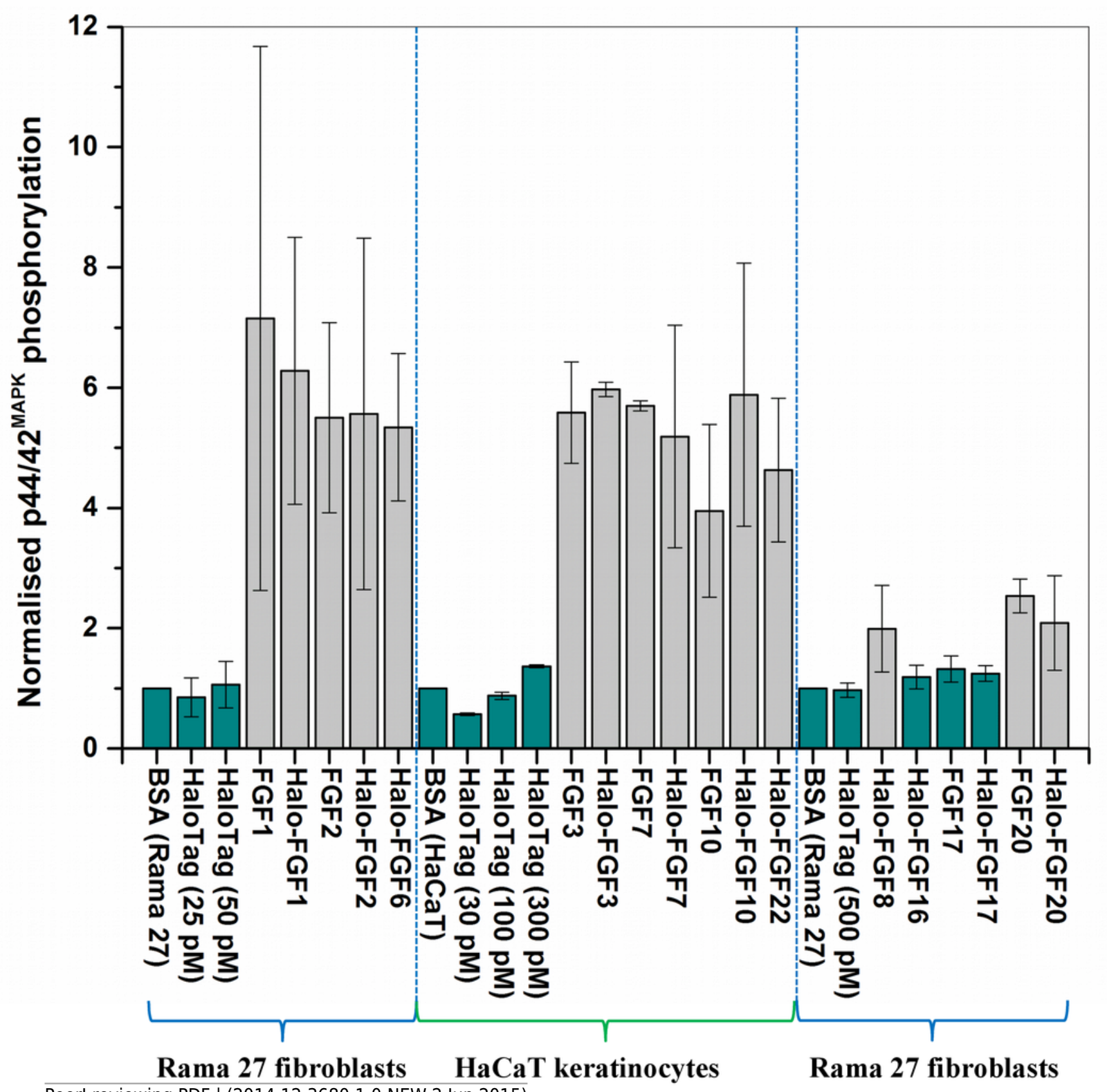




\section{Table $\mathbf{1}_{\text {(on next page) }}$}

Peptide sequences of FGFs, the N-terminal HisTag constructs and the N-terminal HaloTag constructs

FGF names, sequences and amino acid numbering are according to the UniProt entry. FGF1 is an N-terminal truncated protein (Ke et al., 1990). FGF2 does not possess a secretory signal sequence, whereas there is no signal peptide recognised in Uniprot for FGF16 and FGF20; consequently full length protein sequence was expressed. For all other FGFs, the protein expressed was without the Uniprot determined secretory signal sequence. FGFx refers any one of the FGFs. TEV cleavage sites are in red. 


\section{PeerJ Reviewing Manuscript}

1

Name

UniProt Residues in mature protein accession

number

FGF1

P05230 16-155

FGF2

P09038-2 1-155

FGF3

P11487 18-239

FGF6 P10767 38-208

$\begin{array}{lll}\text { FGF7 } & \text { P21781 } & 32-194\end{array}$

FGF8b P55075-3 23-215

FGF10 O15520 38-208

$\begin{array}{lll}\text { FGF16 } & \text { O43320 } & 1-207\end{array}$

FGF17 O60258-1 23-216

$\begin{array}{lll}\text { FGF20 } & \text { Q9NP95 } & 1-211\end{array}$

$\begin{array}{lll}\text { FGF22 } & \text { Q9HCT0 23-170 }\end{array}$

HisTag terminus $\quad \sim \quad$ MKHHHHHHPMSDYDIPTTENLYFQGA-[FGFx]

(pET-M11)

HaloTag and $\quad \sim \quad$ MPEIGTGFPFDPHYVEVLGERMHYVDVGPRDGTPVLFLHG TEV site to NPTSSYVWRNI I PHVAPTHRCIAPDLIGMGKSDKPDLGYF FDDHVRFMDAFIEALGLEEVVLVIHDWGSALGFHWAKRNP ERVKGIAFMEFIRPIPTWDEWPEFARETFQAFRTTDVGRK LIIDQNVF IEGTLPMGVVRPLTEVEMDHYREPFLNPVDRE PLWRFPNELPIAGEPANIVALVEEYMDWLHOSPVPKLLFW GTPGVLIPPAEAARLAKSLPNCKAVDIGPGLNLLQEDNPD LIGSEIARWLSTLEISGEPTTEDLYFQS-[FGFX]

2 
Table 2 (on next page)

Concentrations of $\mathrm{NaCl}$ in $50 \mathrm{mM}$ Tris-Cl buffer (pH 7.4) used for heparin affinity chromatography of FGFs

[ $\mathrm{NaCl}]$ for lysate is the concentration of $\mathrm{NaCl}$ in the sample applied to the column. 


\section{PeerJ Reviewing Manuscript}

1 Name $\quad[\mathrm{NaCl}]$ for lysate $(\mathrm{M}) \quad[\mathrm{NaCl}]$ for wash $(\mathrm{M}) \quad[\mathrm{NaCl}]$ for elution $(\mathrm{M})$

$\begin{array}{llll}\text { FGF1 } & 0.6 & 0.6 & 1.0 \\ \text { FGF2 } & 0.6 & 0.6 & 1.5 \\ \text { FGF3 } & 0.3 & 0.6 & 1.0 \\ \text { FGF6 } & 0.3 & 0.4 & 1.0 \\ \text { FGF7 } & 0.3 & 0.3 & 1.0 \\ \text { FGF8 } & 0.6 & 0.6 & 1.5 \\ \text { FGF10 } & 0.6 & 0.6 & 1.0 \\ \text { FGF16 } & 0.3 & 0.4 & 1.0 \\ \text { FGF17 } & 0.6 & 0.6 & 1.0 \\ \text { FGF20 } & 0.3 & 0.4 & 1.0 \\ \text { FGF22 } & 0.6 & 0.8 & 1.5\end{array}$

2 
Table 3 (on next page)

Summary of the molecular sizes and yields of His-FGFs and Halo-FGFs

The molecular weight of the proteins was calculated from their amino acid sequence. The concentrations and volumes of His-FGFs and Halo-FGFs recovered from heparin affinity chromatography were measured. The impurities identified by SDS-PAGE were quantified using ImageJ relative to the band corresponding to His-FGF and to Halo-FGF and the amount of protein in the eluate from heparin chromatography adjusted accordingly, to provide an estimate of the yield. ${ }^{1}$ Not detected. Insufficient soluble protein for reliable quantification. 


\section{PeerJ Reviewing Manuscript}

1

\begin{tabular}{lllll} 
FGFs & \multicolumn{2}{l}{ Molecular Weight $(\mathrm{kDa})$} & \multicolumn{2}{l}{ Yield $(\mathrm{mg} / \mathrm{L})$} \\
& HisTag & HaloTag & HisTag & HaloTag \\
FGF1 & 19.1 & 50.9 & 14 & 16 \\
FGF2 & 17.3 & 52.2 & 2.5 & 11 \\
& No Tag & & No Tag & \\
FGF3 & 28.2 & 60.0 & 0.5 & 11 \\
FGF6 & 22.3 & 54.1 & n.d. ${ }^{1}$ & 27 \\
FGF7 & 22.2 & 54.0 & 0.6 & 5.6 \\
FGF8 & 25.7 & 57.5 & n.d. $^{1}$ & 1.7 \\
FGF10 & 22.7 & 54.5 & 7.7 $^{1}$ & 9.3 \\
FGF16 & 26.9 & 58.7 & n.d. $^{1}$ & 1.0 \\
FGF17 & 25.8 & 57.6 & n.d. $^{1}$ & 1.5 \\
FGF20 & 26.9 & 58.6 & n.d. $^{1}$ & 10 \\
FGF22 & 20.5 & 52.3 & n.d. $^{1}$ & 2.0
\end{tabular}

2 